\title{
Supporting Reflection on Dementia Care using Proximity Sensors
}

\author{
Lars Müller, Mark Sonnentag, Stephan Heuer \\ Embedded Systems and Sensors Engineering \\ FZI Research Center for Information Technology \\ Karlsruhe, Germany \\ \{lmueller, sonnentag, heuer $\} @$ fzi.de
}

\begin{abstract}
Dementia care requires care home staff to constantly balance daily duties and ad hoc demands. Reflection on the resulting patterns could help carers to improve their care practices. In this paper we describe the evaluation of new low-power proximity sensors in a care home to track and measure these patterns. Carers and residents wear sensors which broadcast a unique ID within a limited range, listen for other sensors, and store all received IDs to measure the co-location of other sensors. Using the sensors, on average $44 \%$ of a carer's shift could be matched to a specific resident or a documentation task. When the results were visualized to carers after the shift, carers could recognize behavior from the raw data and started to discuss care practices. The added value was more important to carers than their privacy.
\end{abstract}

\section{INTRODUCTION}

Providing care to elderly residents that suffer from dementia is a complex task that is continuously challenging staff in care homes. Work tasks, although planned, have to be constantly adapted according to residents' demands. The resulting high staff turnover impacts the care quality. According to the National Care Forum, 30\% of their carers left in 2012 within 1 year and $56.7 \%$ within 2 years [1]. Improving the training on the job is essential to reduce these rates. Reflecting on their work may help carers to learn from their experiences and optimize their work practices to account for the specific needs of the residents. Reflective learning [2] refers to "those intellectual and affective activities in which individuals engage to explore their experiences in order to lead to new understandings and appreciations".

The standard method to evaluate care practices are observational frameworks such as Dementia Care Mapping (DCM) [3] and the Short Observational Framework for Inspection 2 (SOFI 2) [4]. These methods define how specially trained personal can observe and document care practices. Although carers will try to improve their behavior, studies have shown that it does not take long for care staff to forget about the observer and to revert to usual patterns of behavior [3]. SOFI 2 is currently used by the Commission for Social Care Inspection in the UK to "capture, in a systematic way, the experience of care for people who use services who would otherwise be unable to communicate this to an inspector" [5]. These inspections aim to improve the quality of care for people with dementia by providing feedback to carers. Observation frameworks gather rich valuable data but the required effort is high. Additional recorded information from existing information systems could support reflection by enriching past experiences. However, the existing documentation in care homes provides only fragmented information about care practices. More efficient documentation methods are required that can measure care practices on a quantitative level to customize scheduling and will warn carers if residents are neglected.

New sensor technologies [6], [7], [8] capture face-to-face interaction based on the co-location of interaction partners. Interaction partners are equipped with sensors that recognize the proximity of other sensors. If two sensors are in a defined proximity distance, the sensors will register an proximity event that hints at a potential social interaction between persons wearing these sensors. The used technologies range from WiFi [7], active RFID [6] to proprietary protocols [8]. These studies analyzed large scale data that anonymizes the underlying participants to mine and visualize social networks [6] or to understand the infection transmission through these networks [9]. Reflection on care practices, however, requires non-anonymized data on a small scale that allows carers to recognize their own activities and patterns. Moreover, the approaches presented above are either not applicable to dementia patients because of the size of the used sensor [8], their aim at reusing smartphones [7] or the fact that they require the deployment of additional access points [6].

This paper argues that similar technology can support carers in their documentation and reflection on care practices by providing quantitative data about daily work. We present an evaluation of new wearable low-power proximity-sensors in a dementia care home. The developed system is based on a decentralized approach that needs no local installations of additional power supplies, coordinating access points or a central server. The conducted study evaluates (i) if the proximity sensors are accepted by carers and residents, (ii) implications of privacy concerns that are triggered by the captured data, and (iii) if the resulting data is sufficiently detailed and easy to understand to actually support the reflective process.

In the following section we describe the developed system before we outline our evaluation approach in a care home. The results are presented, discussed and the main contributions summarized.

\section{PROXIMITY SENSORS}

The developed system is based on a distributed sensing approach similar to [6], [7], [8]. Figure 1 depicts an example setup of 4 sensors worn by 3 residents and 1 carer. Resident 1 and carer A are in proximity. Hence, we assume that carer $\mathrm{A}$ is providing care to resident 1 . Although resident 2 is very 
close, she is, unlike resident 2 , not within proximity distance. However, if carer A starts to walk, there will probably be a short period of proximity between resident 2 and carer A. The resulting patterns of co-location and assumed care activities can be captured without a localization of each participant.

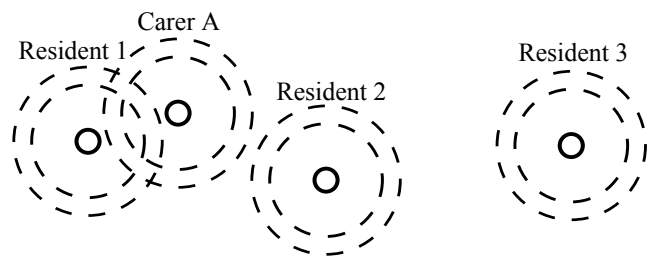

Fig. 1. Schematic example of the proximity between 3 residents and 1 carer

The system design aimed at avoiding the installation of additional infrastructure in the care home. Moreover, the sensors should be as small as possible to minimize interference with care routines. The programmable Chronos eZ430 wristwatch was chosen as the hardware platform, because it is small, includes a low power radio, and can be programmed. We developed an alternative badge format shown in figure 2, because the watch format does not comply with regulations in the care home. A proximity application was installed on each sensor that captures and stores the proximity of other sensors. The data can be later read using a management software.

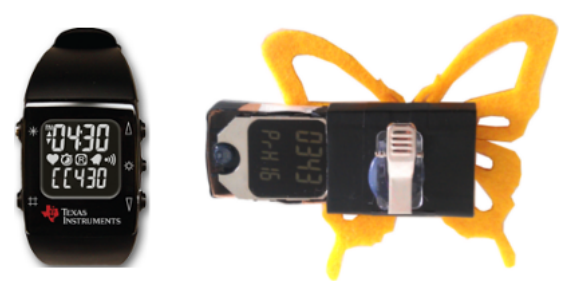

Fig. 2. Chronos eZ430 watch and the wearable badge

The developed proximity application uses the low-power radio module of the Chronos. Each sensor broadcasts every 10 seconds a unique sensor ID in multiple packets. All other sensors are listening for these packets. Proximity between two sensors is detected based on the fact that a radio unit has a physically limited range as shown in figure 1 . The range of the radio module limits the range to slightly beyond the desired proximity distance - the outer circle around each sensor depicted in figure 1. A packet filter reduces undesired false positives to recognize proximity only if sensors are within the desired proximity distance - the inner circles depicted in figure 1. If at least 9 out of 10 packets are received, proximity will be recognized.

The communication between sensors is asynchronous. Hence, the key to optimize the power consumption is the efficient timing of send, listen and sleep phases. A simple unsynchronized media access control was devised and tailored to the specifics of the underlying hardware. Sensors will sleep most of the time to save energy but will check regularly if another sensor is broadcasting. During such a check, the radio module remains in the receive state long enough to receive one packet of a broadcasting sensor in the radio range. If the radio module receives at least one packet, the sensor will stay in the receive state long enough to receive at least 10 packets. Every 10 seconds sensors broadcast their ID for a certain timespan. If sensors attempt to broadcast their proximity packets at the same time, both sensors will sleep for a random number of wait intervals before sending again. In result all timing parameters depend on each other and the underlying hardware.

All data is stored on the sensor and can be analyzed after the shift or the study. The system is intended to be used for a week or even shorter time spans in a care homes, similar to observation frameworks such as SOFI 2 [4]. The sensors were tested using a new coin cell with a capacity of $230 \mathrm{mAh}$. Two sensors were placed 1.5 meter apart to ensure a constant exchange of packets. We measured a maximum runtime of 180 hours. In $98 \%$ of the 10 second intervals both sensors triggered a proximity event. In the study the recognition rate will be lower, because bodies shield the signal and movement will lead to asymmetries in the recognition.

\section{Evaluation IN A CARE Home}

The developed prototype was used as a probe in a care home in the UK that is specialized in dementia care. We collected a first set of data from the target audience in their daily work and mirrored it back to them after the shift. The underlying hypothesis was that carers are able to understand and interpret the data. Hence, visualizations of the data can trigger reflection about critical events.

Observers were not allowed during the study. Nevertheless, the focus of this study was the acceptance and usefulness of the proposed system for carers. Both can be deduced from the captured data and concluding interviews. Are carers willing to distribute and wear sensors for a limited time span? Can the data provide sufficient insights for carers? Which insights can be gained by further analyzing the data?

\section{A. Setting and Method}

The selected care home splits residents by the current state of their disease into 4 groups that are spatially separated into wards. The monitored ward contains single sleeping rooms of residents and two common rooms. If residents are able to leave their bed, they will spend most of the time in the two common rooms; either sitting at one of tables or wandering around. Carers documented their work using a laptop computer at a table in one of the common rooms.

This study was conducted with residents in an advanced stage of dementia that is characterized by a loss of orientation, and by confusing past memories and current activities. However, these residents are still able to act on their own and some of them are still mobile. Due to their illness, residents can react in an unpredictable manner to daily tasks. These incidents of challenging behavior can be understood by reflecting on the residents' life history and the specific reaction.

All carers (3-4 individuals) and residents (9 individuals) on the ward were equipped with proximity sensors during three morning shifts. After discussion with experienced carers, the minimal distance to trigger a proximity event was set to $1.5-2 \mathrm{~m}$. An additional sensor was placed at the laptop computer that was used for documentation tasks. The sensors were disguised with soft and colorful material in the form 
of a brooch. Carers expected that residents will cooperate, if everybody on the ward wears the same kind of colorful badge. Residents remained on the ward during the study. All Carers, except one, worked only on this ward. Hence, the captured data provides a complete picture of the daily work activities of carers and the care received by the residents.

All participants had to sign a consent form to take part in the study. The carers distributed the sensors on 3 consecutive mornings to residents and among each other. The morning shift started at 7:00 and ended at 14:00. After the shift the raw data was visualized using the UnisensViewer [10] and shown to carers. Figure 3 depicts a screenshot of example data shown to carers. In short interviews carers were asked to explain their own raw data, e.g. long periods of documentation or providing care to multiple residents at the same time. This interview was the only possibility to verify the recorded data.

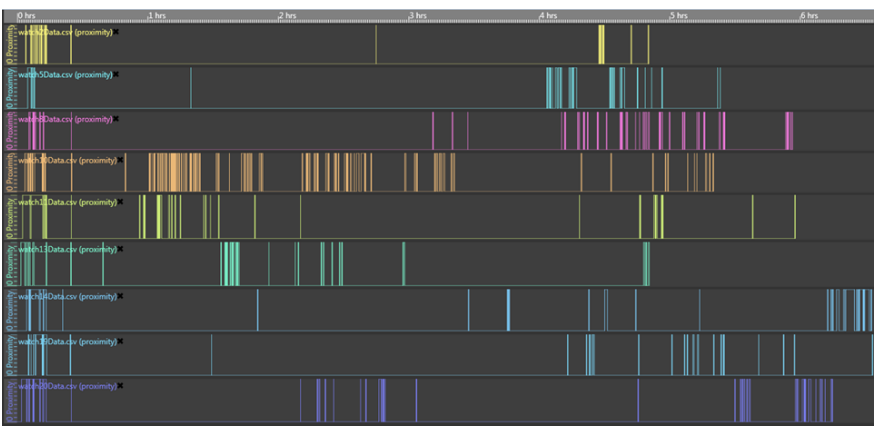

Fig. 3. Example of raw data as shown in the UnisensViewer [10] to carers after the shift. Each row shows a resident and each colored bar in a row indicates proximity to this resident.

\section{B. Results}

15 sensors were used during the 3 days. 2 sensors failed on the first day and 1 on the second day due to residents pulling at them. The sensors were initially attached at the chest of residents and carers. However, residents were confused by this new item and grabbed for them. Therefore, the carers decided to place the sensors at the hip or under a shirt. A total of 41 successful measurements and 290 hours of data were recorded.

Figure 4 illustrates the time shares of carers' shifts that were spent in the proximity of a resident or the documentation laptop. On average $44 \%$ of a carer's shift was captured by at least one sensor and could be assigned to a resident or the laptop. Carer 5 has a lower time share because this carer worked only part time on the monitored ward. The remaining $56 \%$ of the time can be explained by carers walking between sensors, caring from a distance or contacts that are shorter than the 10 second sampling interval.

The time one carer provided to a single resident varies significantly. The differences between residents are visible in the raw data illustrated in figure 3. Some residents had only a few short contacts, e.g. the data shown in the first row. Other rows show unusually long and frequent contacts, e.g. the fourth row. Interview partners confirmed the correctness of the recorded data, but had expected more contacts. They felt that they were busy with residents all day. Although the data was shown in an anonymized manner, carers immediately recognized individual residents by their data: "This must be

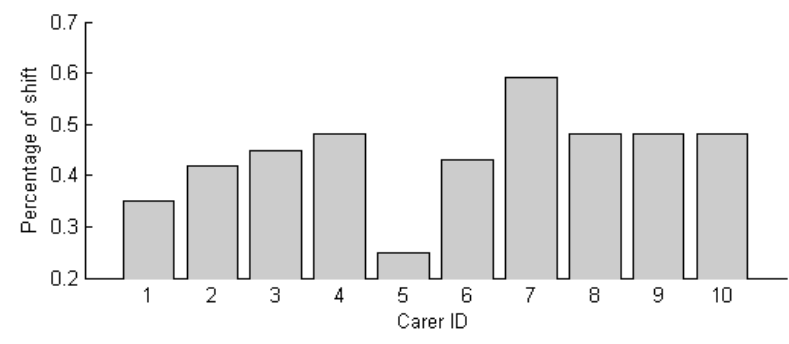

Fig. 4. Recognized proximity of a carer to at least one resident or the laptop during the shift

[name of a resident]"(pointing at a row). Carers explained that one resident with few contacts stayed in bed during the majority of the shift. Unusually long contacts of residents were caused by residents sitting next to a carer who worked on documentation tasks. These reactions prove that the data reflects the actual workday, and the raw data can be understood by carers. However, this raises new concerns regarding the anonymization of recorded data.

Documentation effort was seen as the most tedious and time-consuming task by all carers. When asked before the study, the carers and the care home manager estimated that 30 minutes of care will result in 10 minutes of documentation. However, the proximity sensors showed that on average only $12 \%$ of the proximity events for a carer are related to the documentation laptop. This percentage varies across carers. A new carer spent only $4 \%$ on documentation, while an experienced carer spent $21 \%$ on documentation. Carers confirmed in the interviews that experienced carers support novices in documentation tasks.

The recorded data was analyzed to generate a report for each carer that contained an overview and a timeline for each day. The timeline shown in figure 5 visualizes one shift of a carer during the study. The carers meet in the morning in the common room to plan the day. Afterwards residents are washed, dressed and brought into the common room, if possible. From 10:00 to 13:00 the majority of residents are in the two common rooms. Multiple contacts between carers and residents are now likely, e.g. when two residents are sitting at the same table. After lunch most residents return to their rooms. This timeline is similar to the data presented in the study as show in figure 3 but the aggregation in 5 minute intervals provides a quick overview.

After the last interview a short group discussion between three carers took place. For instance, carers discussed whether attendance to a resident will be influenced, if the resident stays in bed all day. As most residents are in the common rooms this might lead to insufficient attention to residents in the sleeping rooms. The captured data was seen as beneficial to understand care practices and discuss possible improvements. Carers could identify time spent with residents and when contacts took place. This allows the identification of typical patterns and reflecting on reasons for resulting time shares.

All participants said the benefits of the system outweigh their privacy concerns. Carers even suggested more critical approaches, e.g. a long term monitoring using the sensors or how this data can be presented to relatives. Only one carer 


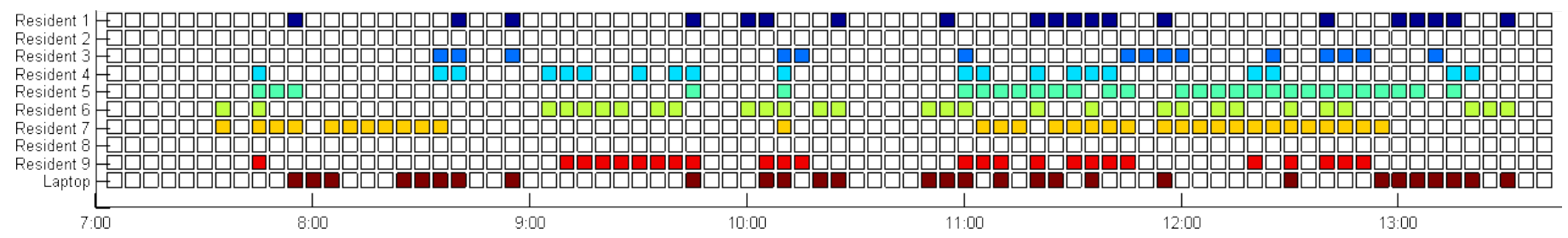

Fig. 5. Timeline of a carer's shift showing the time spent in the proximity of a resident within 5 minute intervals.

voiced privacy concerns, when asked for it. After a detailed description of the system, these concerns could be resolved. Whether this positive attitude towards the system is specific to this care home or the specific setting in the UK, has to be tested in further studies.

The data was further analyzed regarding the symmetry of recorded contacts. If sensor A is within proximity of sensor B, both sensors should record a proximity event. Due to the asynchronous recording and constantly changing proximity, small differences have been expected. In this study, the recorded proximity between two sensors differed up to $20 \%$.

\section{Discussion}

The proximity sensor technology has shown a high potential to support reflective learning by carers, but the amount and quality of the collected data raises privacy concerns. For instance, figure 5 shows a break of the carer between 10:25 and 10:45. If the raw data was available to the management, details of the daily work could be monitored; including the duration of breaks and social interaction between care staff. In consequence, users and management should not have access to raw data. Moreover, we deliberately decided not to transfer the proximity data automatically to a central server. A user wearing a sensor can delete the data before it is read. Furthermore, each participant can decide not to wear the sensor and hence becomes completely invisible for the system. Anonymization of the data is possible but will reduce the usefulness of the data for reflection. In consequence the data should not leave the care home. The opportunities for the management to abuse the system have to be limited but cannot be eliminated completely, because technology can solve only a small part of the privacy challenge.

Nevertheless, the feedback in the care home was overwhelmingly positive. It was surprising how much insights carers gained from the raw data. Carers did not only recognize their timeline but started to reflect and discuss their behavior. Moreover, own ideas were developed on how to use the system. The carers brought up several challenges that need to be addressed. The sensors need a more stable and water proof casing to survive the challenges in a care home. All buttons should be removed or deactivated. Hence, the data transfer from sensors has to be fully automatic. The analysis of the data can be improved to shed light on specific care practices. As task and non-task related proximity events cannot be distinguished, a connection to the official documentation system would be beneficial.

The developed sensor application provided a starting point for our study. Nevertheless, active RFID technology, as in the SocioPatterns project [6], may lower the energy consumption and reduce the maintenance effort. However, we would have to change the underlying hardware of [6] to store the data on the device and eliminate the required access point infrastructure.

\section{CONCLUSiON}

We have presented an approach to turn the eZ430 Chronos hardware into a proximity sensor and described the evaluation of the sensors in a care home. The developed system captures proximity for up to 180 hours powered by a single coin cell. The captured data was useful to spur discussion between carers and trigger collaborative reflection. Especially the time spent on documentation was surprising to care staff. Carers said that these benefits outweigh privacy concerns. Nevertheless, privacy is a key aspect for this kind of application and further research is necessary.

\section{ACKNOWLEDGEMENT}

The project "MIRROR - Reflective learning at work" is funded under the FP7 of the European Commission (project number 257617).

\section{REFERENCES}

[1] "Personnel statistics report 2012 - A Survey of NCF Member Organisations," The National Care Forum, Tech. Rep., 2012.

[2] D. Boud, R. Keogh, and D. Walker, Reflection: Turning Experience into Learning. New York: Routledge Falmer, 1985, ch. Promoting Reflection in Learning: a Model., pp. 18-40.

[3] D. Brooker and C. Surr, "Dementia care mapping (dcm): initial validation of dem 8 in uk field trial," International Journal of Geriatric Psychiatry, vol. 21, no. 11, pp. 1018-1025, 2006.

[4] Short observational framework for inspection 2. [Online]. Available: http://www.brad.ac.uk/health/dementia/dcm/sofi/

[5] D. Brooker and C. Surr, See me, not just the dementia: Understanding peoples' experiences of living in a care home., CSCI, Ed. London: Commission for Social Care Inspection, 2008.

[6] C. Cattuto, W. Van den Broeck, A. Barrat, V. Colizza, J. Pinton, and A. Vespignani, "Dynamics of person-to-person interactions from distributed rfid sensor networks," PLOS ONE, vol. 5, no. 7, p. e11596, 072010.

[7] A. Matic, V. Osmani, A. Maxhuni, and O. Mayora, "Multi-modal mobile sensing of social interactions," in Pervasive Computing Technologies for Healthcare (PervasiveHealth), 2012 6th International Conference on, may 2012, pp. $105-114$.

[8] D. O. Olguin and A. Pentland, "Sensor-based organisational design and engineering," International Journal of Organisational Design and Engineering (IJODE), 2010.

[9] L. Isella, M. Romano, A. Barrat, C. Cattuto, V. Colizza, W. Van den Broeck, F. Gesualdo, E. Pandolfi, L. Rav, C. Rizzo, and A. E. Tozzi, "Close encounters in a pediatric ward: Measuring face-to-face proximity and mixing patterns with wearable sensors," PLOS ONE, vol. 6, no. 2, p. e17144, 022011.

[10] Unisens - a universal data format for multi sensor data. [Online]. Available: http://www.unisens.org 